In the book "Operator theory and Applications", Fields Institute Communications vol. 25, AMS, Providence, 2000, pp. 457-472. (Ed.A.G.Ramm, P.N.Shivakumar, A.V.Strauss).

\title{
Existence and uniqueness of the scattering solutions in the exterior of rough domains
}

\author{
Alexander G. Ramm \\ Department of Mathematics \\ Kansas State University \\ Manhattan, KS 66506-2602, USA. \\ Email: ramm@math.ksu.edu \\ Marco Sammartino \\ Dipartimento di Matematica, \\ Via Archirafi 34, 90123 \\ Palermo, Italy. \\ E-mail: marco@dipmat.math.unipa.it
}

\begin{abstract}
A simple and short proof is given for the existence and uniqueness of the solution to the obstacle scattering problem under weak smoothness assumptions on the obstacle. The Robin, Neumann and Dirichlet boundary conditions are considered.
\end{abstract}

\section{Introduction}

In this paper we study the scattering problem in the exterior of a rough bounded domain. This problem was investigated in [9], where it was assumed that the potential had compact support. The goal of this paper is to relax this hypothesis and to give a simple and general method of proof which is simpler than the earlier known. The assumptions on the coefficients of the differential operator are also relaxed. We prove the results, similar to those in [9], assuming that the potential decays at infinity at the power rate which depends on the space dimension. We discuss the 3 -D case $(n=3)$, but the arguments are similar in the $n$-dimensional case. By this reason in many places we keep $n$ in the formulation of the results and assumptions. Estimates (3.18), (3.19), (4.1), (4.2) and (4.16) are given for $n=3$.

The assumptions on the smoothness of the domain are minimal and include all the previously studied cases and probably all of the cases of interest in applications.

The plan of the paper is as follows. In the rest of this Section we introduce some notations, the function spaces we work with, and give the statement of the scattering problem. In Section 2 we prove the uniqueness of the solution of the scattering problem. In Section 3 we prove the existence of the solution assuming that the potential has compact support. In Section 4 we assume a power decay rate of the potential and prove the existence of the scattering solution.

Our results are stated and proved for the broader class of domains than in the literature, see e.g. [6], [7] and [13]. In particular Lipschitz domains form a proper

1991 Mathematics Subject Classification. Primary 35P25, Secondary 81F05.

Key words and phrases. scattering problem, obstacle scattering, rough domains. 
subset in the class of domains we study. An inverse obstacle scattering problem in a class of rough domains was studied in [11]. See also [8] for Maxwell's equations in rough domains.

The basic ideas of our method are simple: first, we prove that the operator of the problem under consideration is selfadjoint. This is done by using the fact that every closed symmetric densely defined semibounded from below quadratic form on a Hilbert space defines a unique selfadjoint operator whose domain is dense in the domain of the quadratic form. The assumptions (1.4) and (1.5) (see below) concerning the roughness of the domain guarantee that the corresponding quadratic form is semibounded from below and closed. The case of the Dirichlet boundary condition is the simplest one, and in this paper we mostly deal with the Robin boundary condition. In the simplest case of the Dirichlet boundary condition no assumptions are needed on the roughness of the obstacle: any compact domain is admissible. The reason is simple: for the compactness of the embedding $H_{0}^{1}\left(D_{R}\right) \rightarrow$ $L^{2}\left(D_{R}\right)$ no assumptions on $D$, except boundedness of $D$ are needed, while for the compactness of the embedding $H^{1}\left(D_{R}\right) \rightarrow L^{2}\left(D_{R}\right)$ (see next section for the notations and condition (1.4) below) some assumptions concerning the roughness of the boundary of $D$ are necessary. Necessary and sufficient conditions for the compactness of the above embedding operator are known [4], [5]. For the Robin boundary condition in addition to (1.4) assumption (1.5) is needed.

Secondly, we prove that the solution to the problem with complex spectral parameter $k^{2}+i \epsilon, \epsilon>0$, converges, as $\epsilon \rightarrow 0$, to the solution of the scattering problem. The convergence holds in suitable local and global norms.

The underlying idea is to use the Fredholm property of the problem in a properly chosen pair of normed spaces.

1.1 Notations and assumptions. The following are the notations used in this paper.

By $D \subset \boldsymbol{R}^{n}$ we denote a bounded domain, $D^{\prime}$ stands for its complement, $D^{\prime}:=$ $\boldsymbol{R}^{n} \backslash D, D_{R}:=D^{\prime} \cap B_{R}, B_{R}$ denotes a ball with radius $R$ such that $B_{R} \supset D$, and $B_{R}^{\prime}$ is the complement of $B_{R}$ in $\boldsymbol{R}^{n}$.

By $a_{i j}(x), x \in D^{\prime}$, we denote the elements of a real-valued symmetric matrix satisfying the following ellipticity condition:

$$
\exists c, C>0 \text { such that } \quad c|t|^{2} \leq a_{i j}(x) t_{i} \bar{t}_{j} \leq C|t|^{2} \quad \forall t \in C^{n}, \forall x \in D^{\prime} .
$$

In (1.1) and below the summation over the repeated indices is understood. One has:

$$
a_{i j}(x)=\delta_{i j} \text { when }|x|>R,
$$

and the coefficients $a_{i j}(x)$ are assumed Lipschitz continuous. This assumption implies the validity of the unique continuation principle for the solutions to elliptic equation (1.8) below.

Let

$$
l u:=-\partial_{j}\left(a_{i j}(x) \partial_{i} u\right) .
$$

Assume

$$
\begin{aligned}
& q(x)=\overline{q(x)}, \quad q(x) \in L_{\mathrm{loc}}^{p}\left(\boldsymbol{R}^{n}\right), \quad p>\frac{n}{2}, \quad q(x) \in L^{\infty}(\tilde{S}), \\
& |q(x)| \leq \frac{c}{\left(1+|x|^{2}\right)^{s / 2}}, \quad|x|>R, \quad s>n, n \geq 3,
\end{aligned}
$$


where $\tilde{S} \subset D^{\prime}$ is a neighborhood of the boundary $S:=\partial D$. The bar stands for complex conjugate. The assumption $s>n$ is weakened in some of the statements of this paper: for example, in Proposition 2.2 below it is assumed that $s>1$. However, the assumption $s>n, n=3$, is used in section 4. In [1] the scattering theory is developed under the assumptions that $a_{i j}=\delta_{i j}$, the obstacle is absent, and $q(x)$ satisfies less restrictive assumptions than (1.3), essentially it is assumed that $s>1$. Therefore it is likely that the results of our paper can be obtained under this weaker assumption. However, much additional technical work is needed to obtain such results. We are emphasizing the methodology of handling rough boundaries in this paper and discussing of the wider class of potentials would lead us astray.

Let

$$
L u:=l u+q(x) u .
$$

Let $S:=\partial D$. Our main assumptions concerning the smoothness of $D$ are:

$$
\text { The embedding } i: H^{1}\left(D_{R}\right) \rightarrow L^{2}\left(D_{R}\right) \quad \text { is compact, }
$$

$$
\text { The embedding } \quad r: H^{1}\left(D_{R}\right) \rightarrow L^{2}(S) \quad \text { is compact, }
$$

where in the definition of $L^{2}(S)$ the integration over $S$ is understood with respect to the $n$-1-dimensional Hausdorff measure [4]. In particular, assumption (1.5) implies that the $n$-1-dimensional Hausdorff measure of $S$ is finite, that $D$ has a finite perimeter (see [4], p.296), and that Gauss-Green formula holds in $D$ for functions in $B V(D)$ space, which consists of functions $u \in L_{l o c}^{1}(D)$ such that $\nabla u$, understood in the sense of distribution theory, is a signed measure (a charge) in $D$. See $[4]$ for the exact formulation of the Gauss-Green formula for such domains and such class of functions.

Let

$$
u_{N}:=\partial_{N} u:=a_{i j}(x) \partial_{i} u N_{j},
$$

where $N_{j}$ is the j-th component of the normal to $S$ pointing into $D$. The definition of the normal for rough domains is not discussed here because in our formulation of the scattering problem (see (1.11)-(1.12) below) the notion of normal is not used. One can find the definition of the normal in the sense of Federer in [4], p.303.

In this paper we do not define the class of rough domains explicitly, but isolate property (1.4) or properties (1.4) and (1.5) as defining properties. In [4] necessary and sufficient conditions on $S$, the boundary of $D$, are given for these properties to hold. In formulas below in which the surface integrals appear, e.g., (1.11), (2.1), (3.1), (3.2), etc, the integration measure $d s$ is the $n$-1-dimensional Hausdorff measure defined, for example in [4] p.37. In [2] it is proved that for rectifiable surfaces (the class of these surfaces is much broader than the class of Lipschitz surfaces) the $n$-1-dimensional Hausdorff measure is equivalent to the measure generated by the elements of the surface area.

Given an $L^{\infty}(S)$ real-valued function $\sigma(s), s \in S$, one defines the operator $\Gamma^{R}$ :

$$
\Gamma^{R} u(s):=u_{N}(s)+\sigma(s) u(s), \quad s \in S .
$$

Introduce the following operators:

$$
\begin{aligned}
& \Gamma^{D} u(s):=u(s), \quad s \in S, \\
& \Gamma^{N} u(s):=u_{N}(s), \quad s \in S .
\end{aligned}
$$


Let $u_{0}:=\exp (i k \alpha \cdot x), \alpha \in S^{n-1}$, where $S^{n-1}$ is the unit sphere in $\mathbf{R}^{\mathbf{n}}, k>0$, and

$$
v:=u-u_{0} .
$$

1.2 Function spaces. Let

$$
(u, v):=\int_{D^{\prime}} u \bar{v} d x
$$

On $H^{1}\left(D^{\prime}\right)$ define the following bilinear form, $[\cdot, \cdot]: H^{1}\left(D^{\prime}\right) \times H^{1}\left(D^{\prime}\right) \rightarrow \boldsymbol{C}$ :

$$
[u, v]:=\int_{D^{\prime}} a_{i j} \partial_{i} u \partial_{j} \bar{v} d x+(u, v) \text {. }
$$

We use the following spaces:

Definition 1.1 The space $L_{\mathrm{loc}}^{2}\left(D^{\prime}\right)$ is the space of functions $f$ such that, $\forall r>$ $R, f \in L^{2}\left(D_{r}\right)$.

Definition 1.2 The space $H_{\mathrm{loc}}^{l}\left(D^{\prime}\right)$ is the space of functions $f$ such that, $\forall r>$ $R, f \in H^{l}\left(D_{r}\right)$, where $H^{l}\left(D_{r}\right)$ is the Sobolev space.

Definition 1.3 The space $H_{0}^{1}\left(D^{\prime}\right)$ is the closure in $H^{1}\left(D^{\prime}\right)$ norm of the space of functions $f \in H^{1}\left(D^{\prime}\right)$ vanishing near $S$. The set of functions which belong to $H_{0}^{1}\left(D_{r}\right)$ for any $r>R$ is denoted by $H_{0 l o c}^{1}\left(D^{\prime}\right)$.

Definition 1.4 The space $\tilde{H}^{1}\left(D^{\prime}\right)$ is the space of functions $f \in H^{1}\left(D^{\prime}\right)$ vanishing near infinity.

Definition 1.5 The space $\tilde{H}_{0}^{1}\left(D^{\prime}\right)$ is the space of functions $f \in H_{0}^{1}\left(D^{\prime}\right)$ vanishing near infinity.

Definition 1.6 The space $H_{s}^{\ell}:=H_{s}^{\ell}\left(D^{\prime}\right), \ell=0,1$, is the space of functions $f \in H_{\mathrm{loc}}^{\ell}\left(D^{\prime}\right)$ with finite weighted $L^{2}\left(D^{\prime}\right)$-norm, for example the norm in $H_{s}^{\ell}$ with $\ell=1$ is defined as follows:

$$
\|f\|_{1, s}^{2}=\int_{D^{\prime}}\left(1+|x|^{2}\right)^{s / 2}\left(|f|^{2}+|\nabla f|^{2}\right) d x<\infty .
$$

1.3 Statement of the problem. We study the following problem:

$$
\begin{aligned}
& L u-k^{2} u=0 \quad \text { in } D^{\prime}, \\
& \Gamma^{X} u=0 \text { in } S, \\
& \lim _{r \rightarrow \infty} \int_{|x|=r}\left|v_{r}-i k v\right|^{2} d s=0,
\end{aligned}
$$

where $v=u-u_{0}, \quad u_{0}=e^{i k \alpha \cdot x}, \alpha \in S^{n-1}$ is given, $k>0$ is fixed, and $X=R$ (the Robin boundary condition), or $X=N$ (the Neumann boundary condition), or $X=D$ (the Dirichlet boundary condition). We mostly discuss the Robin boundary condition. The other cases can be treated similarly. The Neumann boundary condition is a particular case of the Robin boundary condition $(\sigma(s) \equiv 0)$. The Dirichlet boundary condition is the simplest: it does not require any smoothness assumptions concerning the boundary $S$, only the boundedness of $D$ is required. For the Neumann boundary condition only assumption (1.4) is needed. For the Robin boundary condition both assumptions (1.4) and (1.5) are used. 
1.4 The weak formulation of the scattering problem. Here we introduce the weak formulation of problem (1.8)-(1.10).

Definition 1.7 We say that $u \in H_{l o c}^{1}\left(D^{\prime}\right) \cap H_{-s}^{1}\left(D^{\prime}\right), s>1$, is a weak solution of the scattering problem (1.8)-(1.10) with $X=R$ (the Robin boundary condition) if

$$
\begin{aligned}
& \int_{D^{\prime}}\left[a_{i j} \partial_{i} u \partial_{j} \bar{\varphi}+\left(q-k^{2}\right) u \bar{\varphi}\right] d x+\int_{S} \sigma u \bar{\varphi} d s=0 \quad \forall \varphi \in \tilde{H}^{1}\left(D^{\prime}\right), \\
& \lim _{r \rightarrow \infty} \int_{|x|=r}\left|v_{r}-i k v\right|^{2} d s=0, \quad \text { where } \quad v=u-u_{0} .
\end{aligned}
$$

Definition 1.8 We say that $u \in H_{0 \text { loc }}^{1}\left(D^{\prime}\right) \cap H_{-s}^{1}\left(D^{\prime}\right), s>1$, is a weak solution of the scattering problem (1.8)-(1.10) with $X=D$ (the Dirichlet boundary condition) if

$$
\begin{aligned}
& \int_{D^{\prime}}\left[a_{i j} \partial_{i} u \partial_{j} \bar{\varphi}+\left(q-k^{2}\right) u \bar{\varphi}\right] d x=0 \quad \forall \varphi \in \tilde{H}_{0}^{1}\left(D^{\prime}\right) \\
& \lim _{r \rightarrow \infty} \int_{|x|=r}\left|v_{r}-i k v\right|^{2} d s=0, \quad \text { where } \quad v=u-u_{0}
\end{aligned}
$$

\section{Uniqueness theorem}

In this section we prove uniqueness of the solution to problem (1.8)-(1.10). We first state the following known results:

Proposition 2.1 ([15], p.227) Suppose $u$ is a solution of Eq. (1.8) vanishing on an open subset of $D^{\prime}$. If $a_{i j}(x)$ are Lipschitz and $q(x) \in L_{l o c}^{p}\left(R^{n}\right), p>\frac{n}{2}, n \geq 3$, then $u \equiv 0$ in $D^{\prime}$.

The above Proposition is called the unique continuation principle.

Proposition 2.2 ([10], p.25) Suppose that (1.2) and (1.3) hold, $s>1$ in (1.3) and $k>0$. If $u \in H_{l o c}^{1}\left(B_{R}^{\prime}\right)$ and

$$
\begin{aligned}
& \int_{B_{R}^{\prime}}\left[\partial_{i} u \partial_{i} \bar{\varphi}+\left(q(x)-k^{2}\right) u \bar{\varphi}\right] d x=0 \quad \forall \varphi \in \tilde{H}_{0}^{1}\left(B_{R}^{\prime}\right), \\
& \lim _{r \rightarrow \infty} \int_{|x|=r}|u|^{2} d s=0
\end{aligned}
$$

then $u \equiv 0$ outside $B_{R}$.

In $[\mathbf{1 0}]$, p.25, it is assumed that $q(x)=0$. In the general case the result follows from a theorem of T.Kato $([\mathbf{3}])$ which says that any solution to equation (1.8) in $B_{R}^{\prime}$, which satisfies $(2.2)$, vanishes in $B_{R}^{\prime}$ if $k>0$ and $|x||q(x)| \rightarrow 0$ as $|x| \rightarrow \infty$. It is not necessary to assume $q$ real-valued in Kato's theorem.

We now prove a Lemma used in the proof of the uniqueness of the solution of the scattering problem.

Lemma 2.1 Suppose $W$ satisfies (1.11). Then

$$
\int_{|x|=r}\left(\bar{W} W_{r}-W \bar{W}_{r}\right) d s=0, \quad r>R .
$$

Proof: Take a cut-off function $h(r) \in C^{\infty}(\boldsymbol{R})$ such that $0 \leq h \leq 1, h(r)=1$ when $r \leq 1 / 2, h(r)=0$ when $r \geq 3 / 2, h$ is monotonically decreasing. In (1.11) take 
$\bar{\varphi}=\bar{W} h\left(\left(|x|-r_{0}\right) / \delta\right)$, with $r_{0}>R$, and $\delta>0$. Then take the complex conjugate and subtract. Using (1.2), one gets:

$$
\int_{D^{\prime}}\left(\bar{W} \partial_{i} W-W \partial_{i} \bar{W}\right) \partial_{i} h\left(\left(|x|-r_{0}\right) / \delta\right) d x=0 .
$$

If one takes the limit $\delta \rightarrow 0$ in the above relation, one gets the desired result. Taking this limit, one uses the interior regularity of $W$ : this regularity is a consequence of equation (1.11) and of the known interior elliptic regularity results.

We now state the main result of this Section.

Theorem 2.1 Suppose $u_{1}$ and $u_{2}$ are the weak solutions of the scattering problem with the Robin (Neumann, Dirichlet) boundary condition. Then $u_{1} \equiv u_{2}$.

Proof: We prove this Theorem in the case of the Robin boundary condition. The cases of the Neumann and the Dirichlet boundary conditions can be treated similarly. Define $W:=u_{1}-u_{2}$. One has:

$$
\begin{aligned}
& \int_{D^{\prime}}\left[a_{i j} \partial_{i} W \partial_{j} \bar{\varphi}+\left(q-k^{2}\right) W \bar{\varphi}\right] d x+\int_{S} \sigma W \bar{\varphi} d s=0 \quad \forall \varphi \in \tilde{H}^{1}\left(D^{\prime}\right), \\
& \lim _{r \rightarrow \infty} \int_{|x|=r}\left|W_{r}-i k W\right|^{2} d s=0 .
\end{aligned}
$$

Equation (2.5) can be written as:

$$
\lim _{r \rightarrow \infty} \int_{|x|=r}\left(\left|W_{r}\right|^{2}+k^{2}|W|^{2}\right) d s+\lim _{r \rightarrow \infty} i k \int_{|x|=r}\left(W_{r} \bar{W}-\bar{W}_{r} W\right) d s=0 .
$$

Since $W$ satisfies (2.4), Lemma 2.1 applies, and the above relation implies

$$
\lim _{r \rightarrow \infty} \int_{|x|=r}|W|^{2} d s=0,
$$

which is condition (2.2) of Proposition 2.2. Moreover, from (2.4) and (1.2) it follows that $W$ satisfies condition (2.1). Therefore Proposition 2.2 implies $W=0$ outside $B_{a}$. Then one applies Proposition 2.1 and concludes that $W \equiv 0$ in $D^{\prime}$. Theorem 2.1 is proved.

We wish to prove the existence of the solution of the scattering problem. Let us reduce the problem to the one for a function which satisfies the radiation condition at infinity.

Take a cut-off function $\zeta \in C^{\infty}(\boldsymbol{R})$, such that $0 \leq \zeta \leq 1, \zeta \equiv 0$ in a neighborhood of $D$ and $\zeta \equiv 1$ outside $B_{r}$ for some $r>R$, and define

$$
w:=u-\zeta u_{0} .
$$

If $u$ solves the scattering problem (1.8)-(1.10), then $w$ solves the following problem:

$$
\begin{aligned}
& L w-k^{2} w=f:=\left(L-k^{2}\right)\left(\zeta u_{0}\right), \\
& \Gamma^{X} w=0, \\
& \lim _{r \rightarrow \infty} \int_{|x|=r}\left|w_{r}-i k w\right|^{2} d s=0 .
\end{aligned}
$$

In Section 3 we prove that the above problem has a solution. Theorem 2.1 implies that this solution is unique. 


\section{Existence for compactly supported potential}

In this Section we prove that the scattering problem (1.8)-(1.10) with a compactly supported potential, $q(x)=0$ if $|x|>a$, has the unique solution. We look for a solution of the form $u=w+\zeta u_{0}$, where $\zeta$ is a cut-off function (defined above formula (2.6)) and $w$ satisfies (2.6)-(2.8). Let us prove that problem (2.6)-(2.8) has a solution.

3.1 Existence for the equation with the absorption. Consider the weak form of the scattering problem:

Let $\varepsilon>0$. Find $w \in H_{l o c}^{1}\left(D^{\prime}\right) \cap H_{-s}^{1}\left(D^{\prime}\right), s>1$, such that:

$$
\int_{D^{\prime}}\left[a_{i j} \partial_{i} w \partial_{j} \bar{\varphi}+\left(q-k^{2}-i \varepsilon\right) w \bar{\varphi}\right] d x+\int_{S} \sigma w \bar{\varphi} d s=(f, \varphi) \quad \forall \varphi \in \tilde{H}^{1}\left(D^{\prime}\right),
$$

In the space $H^{1}\left(D^{\prime}\right) \subset L^{2}\left(D^{\prime}\right)$ consider the following bilinear form:

$$
B_{\gamma}[u, v]:=[u, v]+\gamma(u, v)+\int_{S} \sigma u \bar{v} d S+(q u, v):=[u, v]_{\gamma}+(q u, v),
$$

where $[u, v]$ is defined in $(1.7)$ and $\gamma>0$ is chosen so large that the form $[u, u]_{\gamma}^{1 / 2}$ defines the norm equivalent to $H^{1}\left(D^{\prime}\right)$. We use here assumption (1.5), which implies that the boundary integral in $(3.2)$ can be estimated by the term $c|| u\left\|_{H^{1}\left(D_{R}\right)}|| v\right\|_{H^{1}\left(D_{R}\right)}$. One could omit assumption (1.5) and change the space in which the solution is sought, to the space with the norm containing the additional term $\|u\|_{L^{2}(S)}$, where the measure used in the definition of $L^{2}(S)$ is the $n$-1-dimensional Hausdorff measure. Then one could use assumption (1.4), do not use assumption (1.5), and assume that $S$ has finite perimeter (see [14]). A set has finite perimeter if the gradient (in the sense of distribution theory) of the characteristic function of this set belongs to the space $B V(D)$. This space was mentioned below formula (1.5) (see also [14], p.152).

If $X=D$ in (1.9), then the term $\int_{S} \sigma u \bar{v} d S$ is absent in the definition of $[u, v]_{\gamma}$ in (3.2) and assumptions (1.4) and (1.5) can be dropped.

If $X=N$ in (1.9), then the term $\int_{S} \sigma u \bar{v} d S$ is also absent in the definition of $[u, v]_{\gamma}$ in (3.2), assumption (1.4) is used and assumption (1.5) is not used.

If $X=R$ in (1.9), then both assumptions (1.4) and (1.5) are used.

Assumption (1.4) allows one to conclude that (3.5) implies (3.12) (see below) in the case of Neumann or Robin boundary conditions.

Assumption (1.5) allows one to conclude that (3.5) implies that the term $\int_{S} \sigma \psi_{n} \bar{\varphi} d S$ converges and can be estimated by the $H^{1}\left(D_{R}^{\prime}\right)$-norm of $\psi_{n}$ for any test function $\varphi$ in (3.14) (see below).

Assuming (1.1)-(1.5), one has:

Lemma 3.1 The form $B_{\gamma}[\cdot, \cdot]$ is continuous in $H^{1}\left(D^{\prime}\right) \times H^{1}\left(D^{\prime}\right)$ and for all sufficiently large $\gamma>0$ there exist $\beta_{j}, j=1,2$, such that

$$
\beta_{1}\|u\|_{1}^{2} \leq B_{\gamma}[u, u] \leq \beta_{2}\|u\|_{1}^{2} \quad \beta_{1}>0 .
$$

Proof: From (1.3), (1.4) and [12] one gets:

$$
\left.\left|\int_{\tilde{S}} q\right| u\right|^{2} d x \mid \leq \nu\|u\|_{H^{1}(\tilde{S})}^{2}+C(\nu)\|u\|_{L^{2}(\tilde{S})}^{2},
$$

for any $\nu>0$, however small. Denote $\tilde{D_{R}}:=D_{R} \backslash \tilde{S}$, and choose $\tilde{S}$ such that the boundary of $\tilde{D_{R}}$ is smooth. One has $\left.\left|\int_{\tilde{D}_{R}} q\right| u\right|^{2} d x \mid \leq c(R)\|u\|_{1}^{2}$ if (1.3) holds. 
Indeed, if $u \in H^{1}\left(\tilde{D}_{R}\right)$, then, by the Sobolev embedding theorem for domains with sufficiently smooth (say, Lipschitz) boundaries, one has $u \in L^{\frac{2 n}{n-2}}\left(\tilde{D}_{R}\right)$. By Hölder's inequality,

$$
\left.\left|\int_{\tilde{D}_{R}} q\right| u\right|^{2} d x \mid \leq\|q\|_{L^{p}\left(\tilde{D}_{R}\right)}\|u\|_{L^{2 p^{\prime}\left(\tilde{D}_{R}\right)}}^{2},
$$

where $p^{\prime}:=\frac{p}{p-1}$. Choose $p^{\prime}<\frac{n}{n-2}$. Then $p>\frac{n}{2}$. If $p>\frac{n}{2}$ and $u \in H^{1}\left(D_{R}\right)$, then $\left.\left|\int_{\tilde{D}_{R}} q\right| u\right|^{2} d x \mid \leq c(R)\|u\|_{1}^{2}$. Moreover, since the embedding $H^{1}\left(\tilde{D}_{R}\right) \rightarrow L^{2 p^{\prime}}\left(\tilde{D}_{R}\right)$ is compact for $p^{\prime}<\frac{n}{n-2}$, it follows from the above arguments, assumptions (1.3) and (1.4), and from $[\mathbf{1 2}]$ that

$$
\left.\left|\int_{D_{R}} q\right| u\right|^{2} d x|\leq \nu| \mid u\left\|_{H^{1}\left(D_{R}\right)}^{2}+C(\nu)\right\| u \|_{L^{2}\left(D_{R}\right)}^{2}
$$

for any $\nu>0$, however small. Assumption (1.5) and [12] imply

$$
\left.\left|\int_{S} \sigma\right| u\right|^{2} d s|\leq \nu| \mid u\left\|_{H^{1}\left(D_{R}\right)}^{2}+C(\nu)\right\| u \|_{L^{2}\left(D_{R}\right)}^{2}
$$

for any $\nu>0$, however small. Lemma 3.1 is proved.

It follows from the above lemma that the norm $\left[B_{\gamma}(u, u)\right]^{1 / 2}$ is equivalent to $H^{1}\left(D^{\prime}\right)$ norm, the form $B_{\gamma}[\cdot, \cdot]$ is closed, symmetric and densely defined in the Hilbert space $H:=L^{2}\left(D^{\prime}\right)$. Therefore this form defines a unique self-adjoint nonnegative operator $L$ in $H=L^{2}\left(D^{\prime}\right)$ with domain dense in $H^{1}\left(D^{\prime}\right)$. The points $z$ with $\Im z \neq 0$ do not belong to its spectrum. Thus problem (3.1), with $\varepsilon>0$ has a unique solution in $H^{1}\left(D^{\prime}\right)$. We have proved the following:

Proposition 3.1 Problem (3.1) has a unique solution $w_{\varepsilon} \in H^{1}\left(D^{\prime}\right)$.

In the proof of the above Proposition we have not used the compactness of the support of the potential $q(x)$ and gave the proof valid for $q(x)$ satisfying (1.3).

3.2 The limiting absorption principle. In the above subsection we have proved that for each $\varepsilon>0$, problem (3.1) admits a solution $w_{\varepsilon}$. In this subsection we shall prove that one can take the limit for $\varepsilon$ going to zero, and get the solution of (2.6)-(2.8).

We first prove the following fundamental Lemma.

Lemma 3.2 Suppose $\psi_{n} \in H_{l o c}^{1}\left(D^{\prime}\right) \cap H_{-s}^{0}\left(D^{\prime}\right)$, with $s>1$, and, in the weak sense,

$$
\begin{aligned}
L \psi_{n}-\left(k^{2}+i \varepsilon_{n}\right) \psi_{n} & =h_{n} \\
\Gamma^{R} \psi_{n} & =0,
\end{aligned}
$$

with $\varepsilon_{n} \downarrow 0, h_{n} \in L^{2}\left(D^{\prime}\right)$ have compact support and $h_{n} \rightarrow h$ in $L^{2}\left(D^{\prime}\right)$, where $h$ has compact support. Moreover suppose

$$
\left\|\psi_{n}\right\|_{0,-s} \leq M
$$

where $M>0$ is a constant independent of $n$. Then there exists a subsequence of $\left\{\psi_{n}\right\}_{n \in \boldsymbol{N}}$, denoted again by $\left\{\psi_{n}\right\}_{n \in \boldsymbol{N}}$, and a $\psi \in H_{\mathrm{loc}}^{2}\left(D^{\prime}\right) \cap H_{-s}^{1}\left(D^{\prime}\right)$, with $s>1$, such that:

$$
\begin{array}{lll}
\psi_{n} \longrightarrow \psi & \text { strongly in } & H_{\text {loc }}^{2}\left(D^{\prime}\right), \\
\psi_{n} \longrightarrow \psi & \text { strongly in } & H_{-s}^{0}\left(D^{\prime}\right)
\end{array}
$$


with $\psi$ solving the limiting problem:

$$
\begin{aligned}
L \psi-k^{2} \psi & =h, \\
\Gamma^{R} \psi & =0 .
\end{aligned}
$$

Moreover

$$
\lim _{r \rightarrow \infty} \int_{|x|=r}\left|\psi_{r}-i k \psi\right|^{2} d s=0
$$

\section{Proof:} $R$.

Step 1: Convergence of $\psi_{n}$ in $L_{\mathrm{loc}}^{2}\left(D^{\prime}\right)$ and weak convergence in $H^{1}\left(D_{r}\right) \forall r>$

Due to (3.5), there exists a subsequence $\psi_{n} \in H_{-s}^{0}\left(D^{\prime}\right)$ which converges weakly in $L_{l o c}^{2}\left(D^{\prime}\right)$ :

$$
\psi_{n} \longrightarrow \psi \quad \text { weakly in } \quad L_{\text {loc }}^{2}\left(D^{\prime}\right) .
$$

Let us now prove that this subsequence $\psi_{n}$ is bounded in $H_{l o c}^{1}\left(D^{\prime}\right)$ uniformly with respect to $n$. If this is proved, then one gets:

$$
\psi_{n} \longrightarrow \psi \quad \text { weakly in } \quad H_{\text {loc }}^{1}\left(D^{\prime}\right), \psi_{n} \longrightarrow \psi \quad \text { strongly in } \quad L^{2}\left(D_{r}\right) \forall r>R \text {, }
$$

for a subsequence, denoted also by $\psi_{n}$. To prove that $\left\|\psi_{n}\right\|_{H^{1}\left(D_{r}\right)}<c(r) \forall r>R$, take in the weak formulation of (3.3), similar to (3.1), the test function $\varphi:=\psi_{n} \beta(x)$, where $\beta(x)$ is a cut-off function, $0 \leq \beta(x) \leq 1, \beta(x)=1$ for $|x|<r, \beta(x)=0$ for $|x|>r+1, r>R$. It then follows from the analog of (3.1) (see (3.13) below) that $\left\|\psi_{n}\right\|_{H^{1}\left(D_{r}\right)}<c(r) \forall r>R$, where $c(r)>0$ is a constant independent of $n$, as claimed.

Similarly one can prove that, if $s>1$ then

$$
\left\|\psi_{n}-\psi\right\|_{H_{-s}^{1}\left(D^{\prime}\right)} \rightarrow 0, \quad n \rightarrow \infty .
$$

This is done as follows: write (3.1) with $w=\psi_{n}$ and subtract from (3.1) with $w=\psi_{m}$. Use (1.1), (3.7), (3.12), (3.16)-(3.18) to get the desired conclusion.

If $\psi_{n}$ is a weak solution of the problem (3.3)-(3.4), one can write:

$$
\begin{gathered}
\int_{D^{\prime}}\left[a_{i j} \partial_{i} \psi_{n} \partial_{j} \bar{\varphi}+\left(q-k^{2}-i \varepsilon_{n}\right) \psi_{n} \bar{\varphi}\right] d x+\int_{S} \sigma \psi_{n} \bar{\varphi} d s=\left(h_{n}, \varphi\right) \\
\forall \varphi \in \tilde{H}^{1}\left(D^{\prime}\right) .
\end{gathered}
$$

Using (3.11) and (3.12) one can pass to the limit in the above equation and get:

$$
\begin{gathered}
\int_{D^{\prime}}\left[a_{i j} \partial_{i} \psi \partial_{j} \bar{\varphi}+\left(q-k^{2}\right) \psi \bar{\varphi}\right] d x+\int_{S} \sigma \psi \bar{\varphi} d s=(h, \varphi) \\
\forall \varphi \in \tilde{H}^{1}\left(D^{\prime}\right) .
\end{gathered}
$$

Step 2: Convergence of $\psi_{n}$ in $H_{\mathrm{loc}}^{2}\left(D^{\prime}\right)$.

By the elliptic regularity, one concludes that $\psi_{n}$ and $\psi$ are in $H_{\text {loc }}^{2}\left(D^{\prime}\right)$. Moreover, due to the density of $\tilde{H}^{1}\left(D^{\prime}\right)$ in $L^{2}\left(D^{\prime}\right)$, it follows from (3.13) and (3.14) that $\psi_{n}$ and $\psi$ satisfy (almost everywhere in $\left.D^{\prime}\right)(3.3)$ and (3.8) respectively.

Subtract (3.8) from (3.3), and use the elliptic estimate to get:

$$
\begin{gathered}
\left\|\psi_{n}-\psi\right\|_{H^{2}\left(D_{1}\right)} \leq c\left(\left\|\psi_{n}-\psi\right\|_{L^{2}\left(D_{2}\right)}+\left\|h_{n}-h\right\|_{L^{2}\left(D_{2}\right)}+\varepsilon_{n}\left\|\psi_{n}\right\|_{L^{2}\left(D_{2}\right)}\right) \\
\forall D_{1} \subset \subset D_{2} \subset \subset D^{\prime},
\end{gathered}
$$

where $c>0$ is a constant which depends on $D_{1}$ and $D_{2}$ but not on $n$. This estimate proves (3.6), since $\varepsilon_{n} \rightarrow 0$ and $\left\|\psi_{n}\right\|_{L^{2}\left(D_{2}\right)}$ is bounded.

Step 3: The representation formula and the radiation condition. 
If $\psi_{n}$ satisfies (3.3), assumption (1.2) holds and $q(x)=0$ for $|x|>R$, then the following representation formula holds:

$$
\psi_{n}(x)=\int_{S_{R}}\left[\psi_{n}(s) \partial_{N} g_{\varepsilon_{n}}(x, s)-g_{\varepsilon_{n}}(x, s) \partial_{N} \psi_{n}(s)\right] d s \quad \text { for } \quad x \in B_{R}^{\prime},
$$

where $g_{\varepsilon_{n}}=\frac{e^{i \sqrt{k^{2}+i \varepsilon_{n}}|x-y|}}{4 \pi|x-y|}$ is the Green function of the operator $\Delta+\left(k^{2}+i \varepsilon_{n}\right)$ and $N$ in (3.16) and below stands for the normal to $S_{R}$ pointing into $B_{R}^{\prime}$. One can pass to the limit in (3.16) (due to the convergence of $\psi_{n}$ in $H_{\text {loc }}^{2}\left(D^{\prime}\right)$ proved in Step 2 ), and get the following representation formula for $\psi$ :

$$
\psi(x)=\int_{S_{R}}\left[\psi(s) \partial_{N} g(x, s)-g(x, s) \partial_{N} \psi(s)\right] d s,
$$

where $g=\frac{e^{i k|x-y|}}{4 \pi|x-y|}$ is the Green function of the operator $\Delta+k^{2}$ in $\boldsymbol{R}^{3}$. Equation (3.17) implies that $\psi$ satisfies the radiation condition (3.10).

Step 4: A uniform estimate of the behavior of $\psi_{n}$ at infinity.

The representation formulas (3.16) for $\psi_{n}$ and (3.17) for $\psi$, imply the following uniform estimate for the behavior of $\psi_{n}$ at infinity $\left(\right.$ in $R^{3}$ ):

$$
\sup _{n}\left(\left|\psi_{n}\right|+\left|\nabla \psi_{n}\right|\right) \leq \frac{c}{|x|} \quad \text { for } \quad x \in B_{R}^{\prime},
$$

where $c>0$ is a constant independent of $x \in B_{R}^{\prime}$. This estimate will be crucial in the next Step. Note that (3.12), (3.18) and the estimate $\left\|\psi_{n}\right\|_{H^{1}\left(D_{r}\right)} \leq c(r) \forall r \geq R$ imply that $\psi \in H_{-s}^{1}\left(D^{\prime}\right), s>1$.

Step 5: Convergence of $\psi_{n}$ in $H_{-s}^{0}\left(D^{\prime}\right)$ and the conclusion of the proof.

To complete the proof of the Lemma we have to prove the convergence property (3.7). Estimate (3.18) and the assumption $s>1$ imply:

$$
\begin{aligned}
& \left\|\psi_{n}-\psi\right\|_{0,-s}^{2}=\int_{D^{\prime}} \frac{\left|\psi_{n}-\psi\right|^{2}}{\left(1+|x|^{2}\right)^{s / 2}} d x \\
& \quad=\int_{B_{R} \cap D^{\prime}} \frac{\left|\psi_{n}-\psi\right|^{2}}{\left(1+|x|^{2}\right)^{s / 2}} d x+\int_{D_{R}^{\prime}} \frac{\left|\psi_{n}-\psi\right|^{2}}{\left(1+|x|^{2}\right)^{s / 2}} d x \\
& \leq \sup _{B_{R} \cap D^{\prime}}\left[\frac{1}{\left(1+|x|^{2}\right)^{s / 2}}\right]\left\|\psi_{n}-\psi\right\|_{L^{2}\left(B_{R} \cap D^{\prime}\right)}^{2}+4 \pi c^{2} \int_{R}^{\infty} \frac{1}{r^{2}} \frac{1}{\left(1+r^{2}\right)^{s / 2}} r^{2} d r \\
& \leq \eta,
\end{aligned}
$$

with $\eta>0$ arbitrarily small. In the last step of the above estimate we have chosen $R$ so that $4 \pi c^{2} \int_{R}^{\infty} d r /\left(1+r^{2}\right)^{s / 2} \leq \eta / 2$, and (3.11) has been used. This proves (3.7) in $\boldsymbol{R}^{3}$. In other space dimensions the proof is analogous.

With the help of Lemma 3.2 we prove the following a priori estimate for the solutions $w_{\varepsilon}$ of the problem (3.1). In estimate (3.20) below we take $0<\varepsilon<1$, but we could take $0<\varepsilon<\varepsilon_{0}$, where $\varepsilon_{0}>0$ is an arbitrary small fixed number.

Proposition 3.2 The solution $w_{\varepsilon}$ of problem (3.1) satisfies the following a priori estimate:

$$
\sup _{0<\varepsilon<1}\left\|w_{\varepsilon}\right\|_{0,-s} \leq c
$$




\section{Proof:}

We prove this Proposition by contradiction. Suppose (3.20) is false. Find $\varepsilon_{n} \downarrow 0$ such that

$$
\left\|w_{\varepsilon_{n}}\right\|_{0,-s} \geq n .
$$

Define $\psi_{n}:=w_{\varepsilon_{n}} /\left\|w_{\varepsilon_{n}}\right\|_{0,-s}$. Clearly $\psi_{n}$ satisfy all the hypotheses of Lemma 3.2 with $h_{n}=f /\left\|w_{\varepsilon_{n}}\right\|_{0,-s}, h_{n} \rightarrow 0$ in $L^{2}\left(D^{\prime}\right)$. Lemma 3.2 yields that $\psi_{n} \rightarrow \psi$ where $\psi$ solves the problem:

$$
\begin{aligned}
& L \psi-k^{2} \psi=0, \\
& \Gamma^{R} \psi=0, . \\
& \lim _{r \rightarrow \infty} \int_{|x|=r}\left|\psi_{r}-i k \psi\right|^{2} d s=0 .
\end{aligned}
$$

By the uniqueness Theorem 2.1 we get $\psi \equiv 0$. But this contradicts to the fact that $\left\|\psi_{n}-\psi\right\|_{0,-s} \rightarrow 0$ and $\left\|\psi_{n}\right\|_{0,-s}=1$. Proposition 3.2 is proved.

If $w_{\varepsilon}$ satisfies (3.20) one can apply the Lemma 3.2 to $\psi_{n}=w_{\varepsilon_{n}}$ for some $\varepsilon_{n} \downarrow 0$, and with $h_{n}=f$. We have therefore proved the existence of a $w$ solving the problem (2.6)-(2.8) and the main result of this Section:

Theorem 3.1 Scattering problem (1.8)-(1.10) with a compactly supported potential $q$ has a unique solution $u$ of the form $u=w+\zeta u_{0}$. Here $w \in H_{\mathrm{loc}}^{2}\left(D^{\prime}\right) \cap$ $H_{-s}^{1}\left(D^{\prime}\right)$, with $s>1$, and $\zeta$ is a function defined above formula (2.6).

\section{Existence for a decaying potential}

In this section we prove that the scattering problem (1.8)-(1.10), with a potential $q \in H_{s}^{1}$ has a solution. We shall prove the existence of the solution in the same way we did for the scattering problem with a compactly supported potential. First we look for a solution $u$ of the form $u=w+\zeta u_{0}$ so that the problem is reduced to $(2.6)-(2.8)$. Then we prove the existence of a unique solution $w_{\varepsilon}$ for scattering problem (3.1). Finally we shall prove that one can take the limit $\varepsilon \downarrow 0$ and get the solution of $(2.6)-(2.8)$.

\subsection{Existence for the equation with absorption.}

Proposition 4.1 Problem (3.1), with $\varepsilon>0$, has a unique solution $w_{\varepsilon} \in$ $H^{1}\left(D^{\prime}\right)$.

The proof of this proposition is the same as the one of Proposition 3.1.

4.2 The limiting absorption principle. In this subsection we prove that the solution $w_{\varepsilon}$ of problem (3.1) converges, as $\varepsilon \downarrow 0$, to the solution of (2.6)-(2.8).

The main step is the proof of Lemma 4.3 below. We first state two Lemmas:

Lemma 4.1 Suppose that $|g(x, y)| \leq c|x-y|^{-1}$, and $|f(x)| \leq c\left(1+|x|^{2}\right)^{-s / 2}$, $x, y \in D^{\prime}$ and $s>3$. Then

$$
\left|\int_{B_{R}^{\prime}} g(x, y) f(y) d y\right|+\left|\int_{B_{R}^{\prime}} \nabla_{x} g(x, y) f(y) d y\right| \leq \frac{c}{1+|x|}, \quad x \in D^{\prime}
$$




\section{Proof:}

Let $r=|y|, \rho=|x|, y=r(\sin \theta \cos \phi, \sin \theta \sin \phi, \cos \theta), u=\cos \theta$, and let $r, \theta$ and $\phi$ be the spherical coordinates. One has

$$
\begin{aligned}
\left|\int_{B_{R}^{\prime}} g(x, y) f(y) d y\right| & \leq \int_{B_{R}^{\prime} \cap\{y:|y| \leq \rho / 2\}} \frac{|f(y)|}{|x-y|} d y+\int_{\{y:|y| \geq \rho / 2\}} \frac{|f(y)|}{|x-y|} d y \\
& \leq \frac{c}{|x|} \int_{B_{R}^{\prime}}|f(y)| d y+c \int_{\{y:|y| \geq \rho / 2\}} \frac{d y}{|x-y|\left(1+|y|^{2}\right)^{s / 2}} \\
& \leq \frac{c}{|x|}+2 \pi c \int_{\rho / 2}^{\infty} d r \frac{r^{2}}{\left(1+r^{2}\right)^{s / 2}} \int_{-1}^{1} \frac{d u}{\left(\rho^{2}+r^{2}-2 r \rho u\right)^{1 / 2}} \\
& \leq \frac{c}{|x|}+c_{1} \int_{\rho / 2}^{\infty} \frac{d r}{\left(1+r^{2}\right)^{\frac{s-2}{2}}} \frac{1}{\min (r, \rho)} \leq \frac{c}{|x|} .
\end{aligned}
$$

The second integral in (4.1) can be estimated similarly.

If $s>2$ in lemma 4.1, then the argument above yields $o(1)$ as $|x| \rightarrow \infty$, in place of $\frac{c}{|x|}$ term. By a similar argument one can prove that if $s>2$ and $g=$ $\frac{e^{i k|x-y|}}{4 \pi|x-y|}$, then the function $h:=\int_{B_{R}^{\prime}} g(x, y) f(y) d y$ satisfies the radiation condition: $|x|\left|\frac{\partial h}{\partial|x|}-i k h\right| \rightarrow 0$ as $|x| \rightarrow \infty$ uniformly in the directions of $x$. This remark does not allow one to replace $s>3$ in the assumption (1.3) by $s>2$. The reason is: if $q$ is not compactly supported, the function $f$ defined by (2.6) contains the term $q(x) \zeta(x) u_{0}$ which decays as $O\left(\left(1+|x|^{2}\right)^{-\frac{s}{2}}\right)$ for large $|x|$. If $2<s<3$, then the argument given in lemma 4.1 is not sufficient for getting estimate (4.1). It is probable that the basic result, Theorem 4.1 below, can be established for $s>2$ in (1.3), but some additional argument is needed for a proof of such a result.

Lemma 4.2 Suppose that $|g(x, y)| \leq c|x-y|^{-1}$ and $|f(x)| \leq c\left(1+|x|^{2}\right)^{-s / 2}$, $x, y \in D^{\prime}$, and $\psi \in H_{-s}^{0}\left(D^{\prime}\right)$ with $s>3$. Then

$$
\left|\int_{B_{R}^{\prime}} g(x, y) f(y) \psi(y) d y\right|+\left|\int_{B_{R}^{\prime}} \nabla_{x} g(x, y) f(y) \psi(y) d y\right| \leq \frac{c}{|x|}, \quad x \in D^{\prime}
$$

\section{Proof:}


Denote $|x|:=\rho,|y|:=r, T(x, y):=|x-y|^{-2}\left(1+|y|^{2}\right)^{-s / 2}$. One has:

$$
\begin{aligned}
& \left|\int_{B_{R}^{\prime}} g(x, y) f(y) \psi(y) d y\right| \\
& \leq\left[\int_{B_{R}^{\prime}}|g(x, y) f(y)|^{2}\left(1+|y|^{2}\right)^{s / 2} d y\right]^{1 / 2}\left[\int_{B_{R}^{\prime}}|\psi(y)|^{2}\left(1+|y|^{2}\right)^{-s / 2} d y\right]^{1 / 2} \\
& \leq c\|\psi\|_{0,-s}\left[\int_{B_{R}^{\prime}} T(x, y) d y\right]^{1 / 2} \\
& \leq c_{1}\left\{\left[\int_{B_{R}^{\prime} \cap\{y:|y| \leq \rho / 2\}} T(x, y) d y\right]^{1 / 2}+\left[\int_{\{y:|y| \geq \rho / 2\}} T(x, y) d y\right]^{1 / 2}\right\} \\
& \leq \frac{c}{|x|}+2 \pi c\left[\int_{\rho / 2}^{\infty} d r \frac{r^{2}}{\left(1+r^{2}\right)^{s / 2}} \int_{-1}^{1} \frac{d u}{\rho^{2}+r^{2}-2 r \rho u}\right]^{1 / 2} \leq \frac{c}{|x|} .
\end{aligned}
$$

The second integral in (4.2) can be estimated similarly.

We can now prove a Lemma analogous to Lemma 3.2 of the previous Section.

Lemma 4.3 Suppose $\psi_{n} \in H^{1}\left(D^{\prime}\right) \cap H_{-s}^{0}\left(D^{\prime}\right)$, with $s>3$, and, in the weak sense,

$$
\begin{aligned}
L \psi_{n}-\left(k^{2}+i \varepsilon_{n}\right) \psi_{n} & =h_{n}, \\
\Gamma^{R} \psi_{n} & =0,
\end{aligned}
$$

where $\varepsilon_{n} \downarrow 0, h_{n} \in L^{2}\left(D^{\prime}\right),\left|h_{n}(x)\right| \leq c\left(1+|x|^{2}\right)^{-s / 2}, s>3$, and $h_{n} \rightarrow h$ in $L^{2}\left(D^{\prime}\right)$, where $|h(x)| \leq c\left(1+|x|^{2}\right)^{-s / 2}$. Moreover, suppose

$$
\left\|\psi_{n}\right\|_{0,-s} \leq M, \quad s>3
$$

where $M$ is a constant independent of $n$. Then there exists a subsequence of $\left\{\psi_{n}\right\}_{n \in \boldsymbol{N}}$, denoted again by $\left\{\psi_{n}\right\}_{n \in \boldsymbol{N}}$, and $a \psi \in H_{\mathrm{loc}}^{2}\left(D^{\prime}\right) \cap H_{-s}^{1}\left(D^{\prime}\right)$, such that:

$$
\begin{array}{lll}
\psi_{n} \longrightarrow \psi & \text { in } & H_{\mathrm{loc}}^{2}\left(D^{\prime}\right), \\
\psi_{n} \longrightarrow \psi & \text { in } & H_{-s}^{0}\left(D^{\prime}\right),
\end{array}
$$

where $\psi$ solves the following problem:

$$
\begin{gathered}
L \psi-k^{2} \psi=h, \\
\Gamma^{R} \psi=0, \\
\lim _{r \rightarrow \infty} \int_{|x|=r}\left|\psi_{r}-i k \psi\right|^{2} d s=0 .
\end{gathered}
$$

\section{Proof:} $r \geq R$.

Step 1: Convergence of $\psi_{n}$ in $L_{\mathrm{loc}}^{2}\left(D^{\prime}\right)$ and weak convergence of $\psi_{n}$ in $H^{1}\left(D_{r}\right)$,

If (4.5) holds, then

$$
\begin{array}{lll}
\psi_{n} \longrightarrow \psi & \text { strongly in } & L_{\text {loc }}^{2}\left(D^{\prime}\right), \\
\psi_{n} \longrightarrow \psi & \text { weakly in } & H^{1}\left(D_{r}\right) \quad \forall r \geq R .
\end{array}
$$

This is proved in Lemma 3.2.

Step 2: Convergence $\psi_{n}$ in $H_{\text {loc }}^{2}\left(D^{\prime}\right)$. 
One has

$$
\psi_{n} \longrightarrow \psi \quad \text { strongly in } H_{\mathrm{loc}}^{2}\left(D^{\prime}\right)
$$

This is proved as in Lemma 3.2.

As in the Section 3, $\psi_{n}$ satisfies (4.3) and (4.4), and $\psi$ satisfies (4.8) and (4.9).

Step 3: The representation formula for $\psi_{n}$ and the radiation condition.

If $\psi_{n}$ satisfies (4.3) then the following representation formula holds for $x \in B_{R}^{\prime}$ :

$$
\begin{aligned}
\psi_{n}(x)= & \int_{S_{R}}\left[\psi_{n}(s) \partial_{N} g_{\varepsilon_{n}}(x, s)-\partial_{N} \psi_{n}(s) g_{\varepsilon_{n}}(x, s)\right] d s \\
& +\int_{B_{R}^{\prime}} g_{\varepsilon_{n}}(x, y) h_{n}(y) d y-\int_{B_{R}^{\prime}} g_{\varepsilon_{n}}(x, y) q(y) \psi_{n}(y) d y,
\end{aligned}
$$

where $N$ in the above formula and below denotes the normal to $S_{R}$ pointing into $B_{R}^{\prime}$. Since $\psi_{n}$ converges in $H_{\mathrm{loc}}^{2}\left(D^{\prime}\right)$ one can pass to the limit in (4.14) and get:

$$
\begin{aligned}
\psi(x)= & \int_{S_{R}}\left[\psi(s) \partial_{N} g(x, s)-\partial_{N} \psi(s) g(x, s)\right] d s \\
& +\int_{B_{R}^{\prime}} g(x, y) h(y) d y-\int_{B_{R}^{\prime}} g(x, y) q(y) \psi(y) d y .
\end{aligned}
$$

Equation (4.15) implies that $\psi$ satisfies the radiation condition.

Step 4: A uniform estimate of the behavior of $\psi_{n}$ at infinity.

As in the case of a compactly supported potential one gets the following estimate for the behavior of $\psi_{n}$ at infinity:

$$
\sup _{n}\left(\left|\psi_{n}\right|+\left|\nabla \psi_{n}\right|\right) \leq \frac{c}{|x|} \quad \text { for } \quad x \in B_{R}^{\prime},
$$

where $c>0$ is a constant independent of $x \in B_{R}^{\prime}$. This follows from (4.14) and (4.15). The additional terms which appear because the potential and the source term are not compactly supported, are estimated in Lemmas 4.1 and 4.2. Estimates (4.12) and (4.16) imply $\psi \in H_{-s}^{1}\left(D^{\prime}\right), s>1$.

Step 5: Convergence of $\psi_{n}$ in $H_{-s}^{0}\left(D^{\prime}\right)$ and the conclusion of the proof.

One proves that $\left\|\psi_{n}-\psi\right\|_{0,-s} \rightarrow 0$ as in Lemma 3.2. This concludes the proof of Lemma 4.3 .

Now one gets the following a priori estimate for the solution $w_{\varepsilon}$ of the problem 3.1 :

Proposition 4.2 The solution of problem (3.1) satisfies the following a priori estimate:

$$
\sup _{0<\varepsilon<1}\left\|w_{\varepsilon}\right\|_{0,-s} \leq c, \quad s>1 \text {. }
$$

The proof is based on Lemma 4.3, and is the same as the proof of Proposition 3.2 .

Let us state the main result of this paper, whose proof is based on the a priori estimate (4.17) and Lemma 4.3:

Theorem 4.1 Assume that conditions (1.1)-(1.5) hold and $\sigma(s)$ is a realvalued $L^{\infty}(S)$ - function. Then the scattering problem (1.8)-(1.10) has a weak solution $u$ of the form $u=w+\zeta u_{0}$, with $w \in H_{\mathrm{loc}}^{2}\left(D^{\prime}\right) \cap H_{-s}^{1}\left(D^{\prime}\right), s>1, w$ satisfies (3.1) and (2.8), $\zeta$ is defined above formula (2.6), and this solution is unique in the above space. 


\section{References}

[1] S.Agmon Spectral properties of Schrödinger operators and scattering theory, Ann. Sc. Norm. Sup. Pisa, 4, N2, (1975), 151-218.

[2] H.Federer Geometric measure theory, Springer Verlag, Berlin, 1969.

[3] T. Kato Growth properties of solutions of the reduced wave equation with a variable coefficient, Comm. Pure and Appl. Math., 12, (1959), 403-425.

[4] V.Mazya, Sobolev spaces, Springer Verlag, New York, 1985.

[5] V.Mazya, S.Poborchi, Differentiable functions on bad domains, World Sci., Singapore, 1997.

[6] D.Mitrea, M.Mitrea, J.Pipher, Vector potential theory on nonsmooth domins and applications to electromagnetic scattering, Jour Fourier Anal. Applic., 3,N2, (1997), 131-192.

[7] M.Mitrea, Boundary value problems and Hardy spaces associated to the Helmholtz equation in Lipschitz domains, J. Math. Anal. Appl., 202, (1996), 819-842.

[8] R.Picard, On a selfadjoint realization of curl in the exterior domains, Math. Zeit., 229, (1998), 319-338.

[9] A.G.Ramm and A.Ruiz, Existence and uniqueness of scattering solutions in non-smooth domains, J.Math.Anal.Appl., 201, (1996), 329-338.

[10] A.G.Ramm, Scattering by obstacles, Reidel, Dordrecht, 1986.

[11] A.G.Ramm, Uniqueness theorems for inverse obstacle scattering problem in Lipschitz domains, Applic. Analysis, 59, (1995), 377-383.

[12] A.G.Ramm, A necessary and sufficient condition for compactness of embedding, Vestnik Leningrad. Univ. ser. Mathem., Mech., Astron., N1, (1963), 150-151.

[13] G. Verchota, Layer potentials and regularity for the Dirichlet problem in Lipschitz domains, Jour. Functional Anal., 59, (1984), 572-611.

[14] A. Volpert, S. Hudjaev, Analysis in classes of discontinuous functions and equations of mathematical physics, Martinus, Dordrecht, 1985.

[15] T. Wolff, A property of measures and an application to unique continuation, Geom. and Funct. Anal. 2, N2, (1992), 225-284. 